\title{
Machine-Free Polymerase Chain Reaction with Triangular Gold and Silver Nanoparticles
}

\author{
Pradeep Kadu, ${ }^{[\mathrm{a}] \#}$ Satyaprakash Pandey, ${ }^{[\mathrm{a}]}$ \# Suditi Neekhra, ${ }^{[\mathrm{a}]}$ Rakesh Kumar ${ }^{[\mathrm{a}]}$, Laxmikant \\ Gadhe,${ }^{[\mathrm{a}]}$ Rohit Srivastava, ${ }^{[\mathrm{a}]}$ Murali Sastry, $\left.{ }^{[\mathrm{b}]}\right]_{*}$ Samir K Maji ${ }^{[\mathrm{a}]} *$ \\ [a] Department of Biosciences and Bioengineering, Indian Institute of Technology Bombay, Powai, Mumbai \\ 400076, India \\ [b] IITB-Monash Research Academy, Academy Building, Indian Institute of Technology Bombay, Powai, \\ Mumbai 400076, India and Department of Materials Engineering and Department of Chemical Engineering, \\ Monash University, Clayton VIC 3800, Australia
}

\section{Experimental Procedures}

Materials. All chemicals and reagents were purchased from commercial suppliers, Hydrogen tetrachlorocuprate trihydrate $\left(\mathrm{HAuCl}_{4} 3 \mathrm{H}_{2} \mathrm{O}\right)$, sodium borohydride $\left(\mathrm{NaBH}_{4}\right)$, Hydrochloric acid ( $\mathrm{HCl} 37 \%)$ were purchased from Merck, India. Poly-sodium styrene sulfonate (PSSS), silver nitrate $\left(\mathrm{AgNO}_{3}\right)$ and ascorbic acid were purchased from Sigma Aldrich, USA.

Synthesis of triangular and spherical Gold Nanoparticles. Triangular gold nanoparticles were synthesized using previously reported methods. ${ }^{[1]}$ Lemongrass plant extract was prepared using $5 \mathrm{gm}$ of thoroughly washed leaves, which were finely cut and boiled with 25 $\mathrm{mL}$ of sterile water for 20 minutes. The extract was filtered using Whatman filter paper 42 and used for further steps. The triangular gold nanoparticles were prepared by reducing $1 \mathrm{~mL}$ of an aqueous solution of $1 \mathrm{mM}$ of $\mathrm{HAuCl}_{4}$ with $40 \mu \mathrm{L}$ of an aliquot of lemongrass extract and the spherical nanoparticles were prepared by reducing $1 \mathrm{~mL}$ of an aqueous solution of 1 $\mathrm{mM}$ of $\mathrm{HAuCl}_{4}$ with $80 \mu \mathrm{L}$ of an aliquot of lemongrass extract. Finally, the volume adjusted to $1100 \mu \mathrm{L}$ with autoclaved M.Q. water. UV visible and NIR spectrum were recorded after 24 hours of reduction reaction by Jasco dual-beam spectrophotometer (model V-570). The particles were centrifuged three times at $6000 \mathrm{rpm}$ after 48 hours of reaction to remove residual lemongrass extract from the reaction mixture. Finally, the volume was adjusted to 1 $\mathrm{mL}$ with sterile water and used for further experiments.

Synthesis of triangular and spherical Silver Nanoparticles. A typical synthesis of silver nanoparticles was done using the seed mediated method as described previously. ${ }^{[2]}$ Silver seed was synthesized by mixing poly(sodium styrene sulfonate) (PSSS; $0.25 \mathrm{~mL}, 500 \mathrm{mg} / \mathrm{L}$ ), trisodium citrate $(5 \mathrm{~mL}, 2.5 \mathrm{mM})$ and aqueous sodium borohydride $\left(\mathrm{NaBH}_{4} ; 0.3 \mathrm{~mL}, 10 \mathrm{mM}\right)$ followed by addition of aqueous silver nitrate $\left(\mathrm{AgNO}_{3} ; 5 \mathrm{~mL}, 0.5 \mathrm{mM}\right)$ with continuous stirring at room temperature. The appearance of yellow color indicates seed synthesis in the solution. This seed is now used for nanoparticle growth. The triangular nanoparticles are produced by mixing $5 \mathrm{~mL}$ distilled water, aqueous ascorbic acid $(75 \mathrm{~mL}, 10 \mathrm{mM})$ and $20 \mu \mathrm{L}$ 
of seed solution, followed by addition of aqueous $\mathrm{AgNO}_{3}(3 \mathrm{~mL}, 0.5 \mathrm{mM})$. While the spherical nanoparticles were synthesized using $30 \mu \mathrm{L}$ of seed solution under similar conditions. The synthesis was completed after three minutes of reaction time and the solution changes from yellow to light blue as surface plasmons of nanoparticles shift towards longitudinal waves. After synthesis, aqueous trisodium citrate $(0.5 \mathrm{~mL}, 25 \mathrm{mM})$ is added to stabilize the particles. UV visible and NIR spectrum were recorded immediately after synthesis by Jasco dual-beam spectrophotometer (model V-570).

Transmission electron microscopy (TEM). The samples were prepared by drop-casting 20 $\mu \mathrm{L}$ of $\mathrm{Ag}$ and $\mathrm{Au}$ NPs on carbon-coated copper grids followed by 10 minutes of incubation at room temperature. The unbounded NPs were washed twice with autoclaved Milli-Q water and the sample was dried under IR lamp for 5 minutes. The grids were subjected to transmission electron microscopy (TEM) imaging TEM (Phillips CM-200) and FEG-TEM (FEI Tecnai) at $200 \mathrm{kV}$ with $6000 \mathrm{X}$, and $27000 \mathrm{X}$ magnifications. Images were recorded using Keen View Soft imaging system (Olympus, Tokyo, Japan).

Stability of the nanoparticle. The photothermal stability of nanoparticles was assessed after performing 35 PCR cycles. A $100 \mu \mathrm{L}$ of aqueous AuTNP and AgTNPs were measured using UV-Vis spectroscopy and TEM imaging before and after $808 \mathrm{~nm}$ laser irradiation. The temperature was recorded at each PCR cycle by digital K-Type thermocouple. TEM images were captured before and after the amplification to determine if any deformity occurred during the thermocycling steps of PCR.

X-ray diffraction (XRD). X-ray diffraction studies were performed with the Rigaku smart lab Japan, $3 \mathrm{~kW}$ X-ray diffractometer. Samples were prepared by drop-casting $100 \mu \mathrm{L}$ of Ag and $\mathrm{Au}$ nanoparticles on thin glass films followed by incubation at room temperature for 2 hours. Thereafter the samples were dried under vacuum overnight at room temperature. Experiments were performed at $\mathrm{CuK} \alpha$ radiation and $40 \mathrm{kV}$ voltages. The wavelength for diffraction studies was $1=1.543 \AA$ with a scanning range of $20-90$ (2q degree). The image files were analyzed using Adxv software (Scripps Research Institute, USA).

X-ray photoelectron spectroscopy (XPS). The X-ray photoelectron spectroscopy (XPS) analysis of synthesized Ag and Au NPs were performed on the ESCA model Axis Supra, UK $\mathrm{X}$-ray photo spectrometer at $2 \times 10^{-9}$ Torr with XPS source of (A1K $\alpha 600 \mathrm{~W}$ ). Samples were prepared by drop-casting $20 \mu \mathrm{L}$ of $\mathrm{Ag}$ and Au nanoparticles on aluminum foil followed by incubation at room temperature for 2 hours. Thereafter the samples were dried under vacuum for overnight at room temperature. The $\mathrm{Au} 4 \mathrm{f}, \mathrm{Ag} 3 \mathrm{~d}$ and $\mathrm{C}$ 1s spectra were recorded with XRay source of $(\mathrm{AlK} \alpha) 600 \mathrm{~W} ; 1436 \mathrm{eV}$ photon energy. The core-level spectra were background corrected with Shirley algorithm and alignment of core level binding energies (BEs) was performed using $\mathrm{C}$ 1s binding energy of $284.7 \mathrm{eV}$. XPS peak fitting and analysis has been done with OriginPro-8 software.

Establishment of temperature variation using gold and silver nanoparticle. A $100 \mu \mathrm{L}$ of aqueous AuTNP and AgTNPs samples at different concentrations (0.2, 0.4, 0.6, 0.8, 1.0, 1.2, 
1.4, 1.6 and $1.8 \mathrm{mM}$ ) was irradiated with 1.2 Ampere, $808 \mathrm{~nm}$ laser. The dynamic temperature was measured using a K-Type thermocouple. Additionally, the experiment was performed with varying NPs concentration as a function of time and different laser powers $(0.6,0.7,0.8,0.9,1.0$ and 1.2 Ampere). The thermal decay profile was acquired by turning off the NIR laser and decay temperature was recorded using K-Type thermocouple.

PCR reaction in presence of gold and silver nanoparticles. The NIR light source for thermal profiling was achieved by irradiating gold and silver nanoparticles with $808 \mathrm{~nm}$ laser by adjusting different laser power. The manual cycles were carried out as follows: denaturation of DNA with a temperature gradient of $90,85,80$ and $70{ }^{\circ} \mathrm{C}$ for $20 \mathrm{sec}$, followed by annealing at $55{ }^{\circ} \mathrm{C}$ for $30 \mathrm{sec}$ and extension at $65{ }^{\circ} \mathrm{C}$ for $60 \mathrm{sec}$. The temperature of photothermal PCR was monitored by switching the laser power. The 1.2, 0.85 and 0.7 Ampere (A) laser power is producing $88-90{ }^{\circ} \mathrm{C}, 67-70{ }^{\circ} \mathrm{C}$ and $55-58{ }^{\circ} \mathrm{C}$ heat respectively. The heat generated by the particles was measured by a digital thermocouple. A typical photothermal PCR has been achieved by mixing A $100 \mu \mathrm{L}$ of the colloidal gold $(1.2 \mathrm{mM})$ /silver nanoparticle $(0.43 \mathrm{mM})$ into the 96 well plate chamber with PCR reagents contained $50 \mathrm{ng}$ of plasmid template, $1 \mu \mathrm{L}$ of $10 \mathrm{X}$ buffer, $200 \mu \mathrm{M}$ dNTPs, $500 \mathrm{nM}$ forward and reverse primers and Taq Polymerase $(5 \mathrm{U} / \mu \mathrm{L})$. The reaction cycles and the steps of photothermal PCR were designed similarly to the conventional PCR reaction conditions and the final products of 25 PCR cycles were visualized onto a $1.2 \%$ Agarose gel and the bands were quantified using ImageJ software.

PCR reaction in the conventional machine and real-time PCR machine. PCR reaction was carried out using a plasmid encoding for mCherry ORF (749 bp) in Veriti 96-well thermal cycler. PCR reactions contained $50 \mathrm{ng}$ of plasmid template, $1 \mu \mathrm{L}$ of $10 \mathrm{X}$ buffer, 200 $\mu \mathrm{M}$ dNTPs, $500 \mathrm{nM}$ forward and reverse primers and Taq Polymerase $(5 \mathrm{U} / \mu \mathrm{L})$. The total volume of the reaction used in the PCR machine was $20 \mu \mathrm{L}$. To compare the total yield from the PCR using laser-nanoparticles PCR, end products were visualized onto a $1.2 \%$ Agarose gel and the bands were quantified using ImageJ software.

To calculate the PCR efficiency in presence of nanoparticles, we employed a range of nanoparticle concentrations $(0.5 \mathrm{nM}$ to $10 \mathrm{nM})$, which were added to the Maxima $\mathrm{SyBr}$ Green PCR reaction (Fermentas) and the mixture was subjected to quantitative real-time PCR (AriaMX, Agilent technologies). The thermal profile used for the reaction was: $90^{\circ} \mathrm{C}$ for 10 minutes, followed by 35 cycles of amplification with $90{ }^{\circ} \mathrm{C}$ for $15 \mathrm{sec}, 60{ }^{\circ} \mathrm{C}$ for $30 \mathrm{sec}, 72$ ${ }^{\circ} \mathrm{C}$ for $60 \mathrm{sec}$ and a final extension of 5 minutes. SYBR Green intercalates between the synthesized double-stranded DNA molecules and emits fluorescence. The curves obtained were analyzed for their threshold fluorescence after subtracting the background fluorescence of the dye. The threshold cycle $(\mathrm{Ct})$ is calculated wherein the fluorescence from the reaction is greater than fluorescence values from a "no amplification control". Lower the Ct value, greater is the efficiency of the reaction. The data was plotted using OriginPro 9.0 software.

UV-melting and CD binding studies. DNA and triangular nanoparticle interactions were performed by UV-melting and CD binding experiments. CD binding study was performed on the JASCO-1500 instrument with the quartz cell with $1 \mathrm{~cm}$ of path length. The scanning 
wavelength was 300 to $200 \mathrm{~nm}$ and the spectra were recorded by averaging 3 accumulations for each sample with a time frame of $80 \mathrm{~nm}$ per minute. The signal observed at 275 maxima and 220 minima and the molar ellipticity $(\Delta \varepsilon)$ are related to ellipticity $\left(\mathrm{M}-1 \mathrm{~cm}^{-1}\right)$. DNA melting was carried out in the presence of calf-thymus (Ct-DNA), at $260 \mathrm{~nm}$ with a temperature range of 40 to $90{ }^{\circ} \mathrm{C}$ with a rate of $2{ }^{\circ} \mathrm{C} /$ minute. The concentration of Ct-DNA was measured by its known extinction coefficient at $260 \mathrm{~nm}\left(\varepsilon=6600 \mathrm{M}^{-1}\right)$. The experiment was performed by titrating Ct-DNA $(600 \mu \mathrm{M})$ with different concentrations ratios of gold and silver nanoparticles ranging from 1:1 to 1:7 (DNA: NPs) in $50 \mathrm{mM}$ Tris $\mathrm{HCl}$ buffer at $\mathrm{pH}$ 7.5. The absorbance spectra were recorded using the Jasco dual-beam spectrophotometer (model V-570) using thermal Peltier MCB-100. 


\section{Supplementary Figures}

A

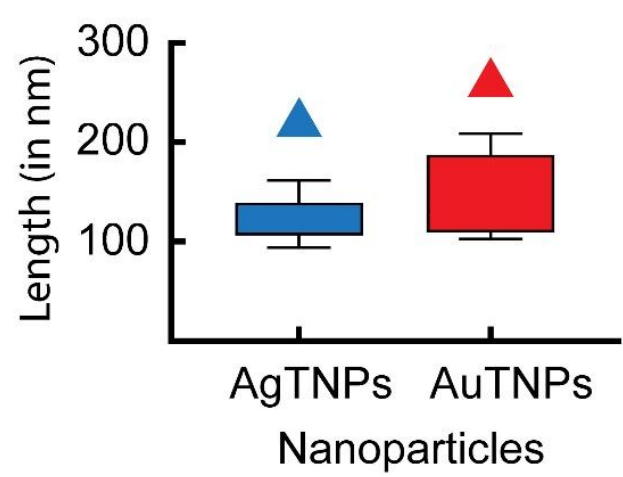

$\mathrm{B}$

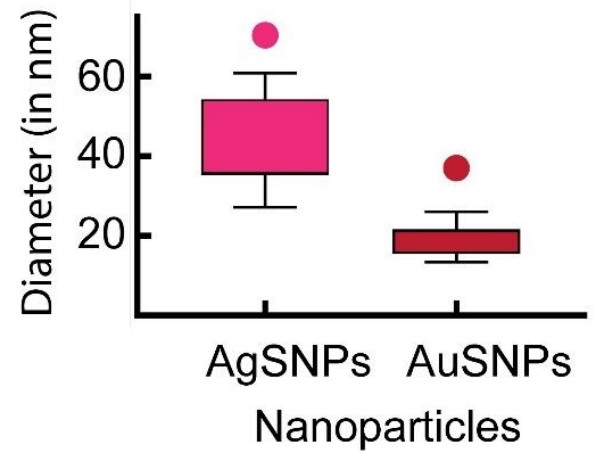

Figure S1. Size of the synthesized nanoparticles. Boxplots denoting the average edge length of TNPs and average diameter of SNPs nanoparticles measured from 100 nanoparticles of each class calculated from transmission electron micrographs. (A) The average size length (edge) of AgTNPs and AuTNPs were $126 \mathrm{~nm}$ (standard deviation $\pm 24 \mathrm{~nm}$ ) and $149 \mathrm{~nm}$ (standard deviation $\pm 45 \mathrm{~nm}$ ), respectively. (B) For spherical nanoparticles, the average diameter of AgSNPs and AuSNPs were $45 \mathrm{~nm}$ (standard deviation $\pm 13.5 \mathrm{~nm}$ ) and $\sim 19 \mathrm{~nm}$ (standard deviation $\pm 5 \mathrm{~nm}$ ), respectively. The image analysis was performed using Image $\mathrm{J}$ software. The data represented are mean \pm standard deviation. 

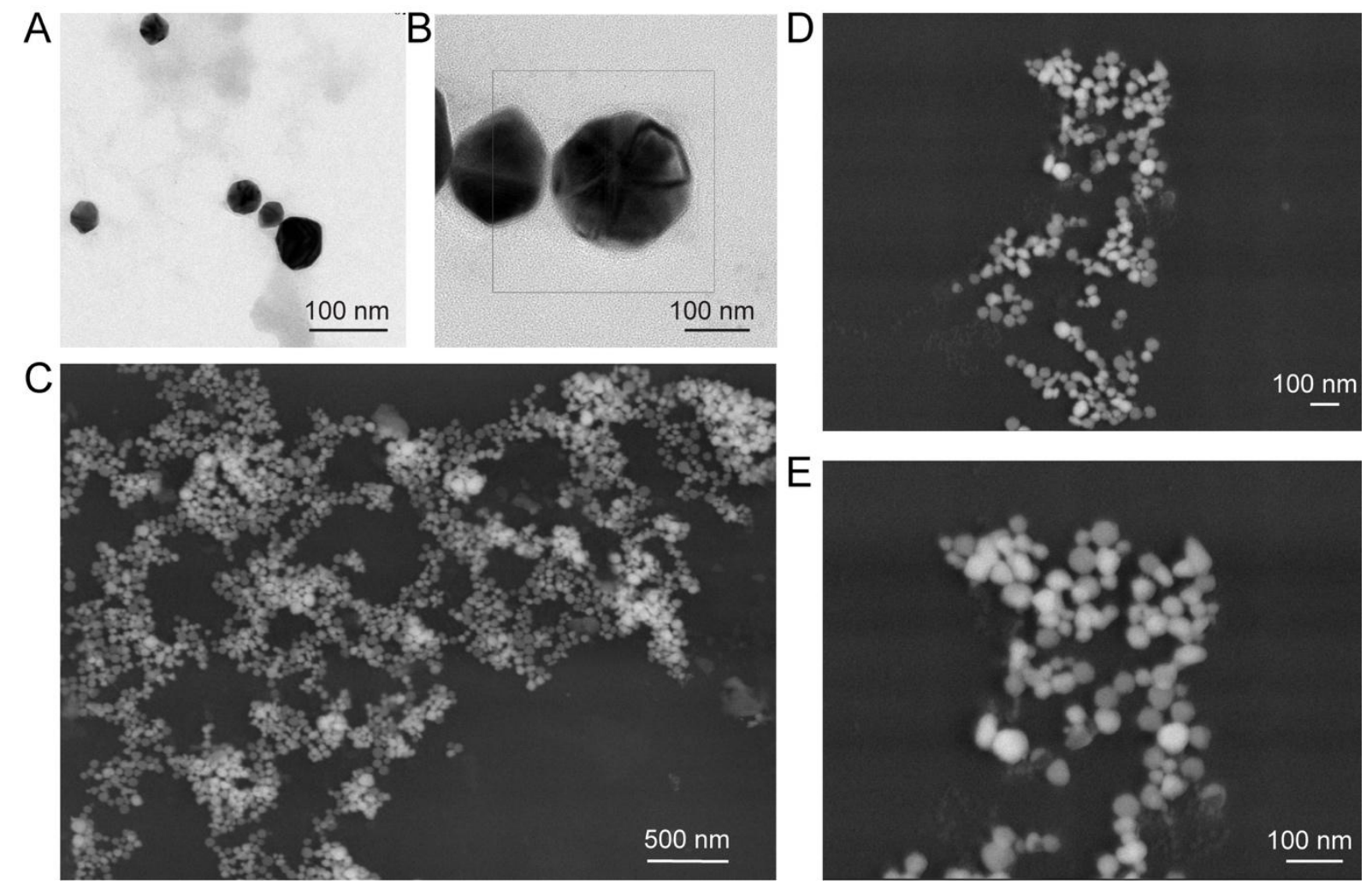

Figure S2. Transmission electron microscopy (TEM) images of spherical silver nanoparticles (AgSNPs) in [A] and [B] showing the quasi-spherical morphology of the spherical silver nanoparticle. Scanning electron microscopy (SEM) images in panel [C] and the highresolution images in panels ([D] and [E]) showing the morphology to be majorly spherical nanoparticles. The scale bars are denoted in the inset. 

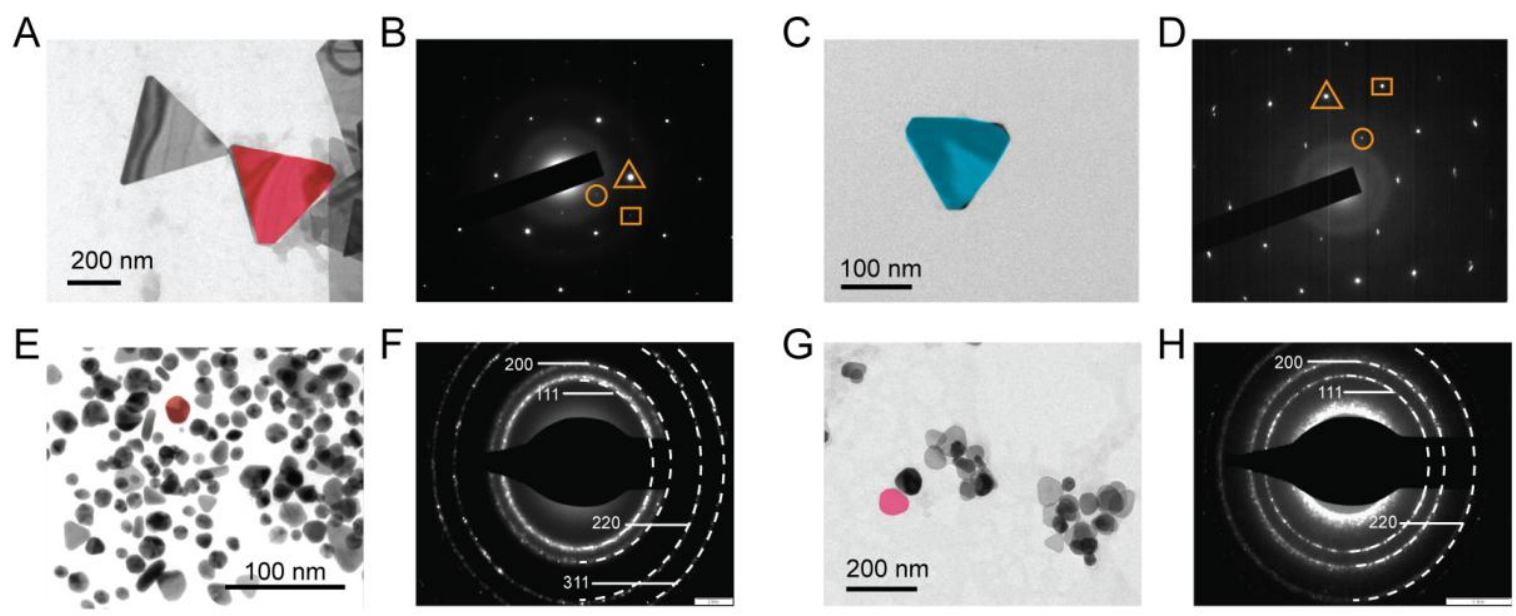

Figure S3. Transmission electron microscope (TEM) images of gold and silver nanoparticles and its corresponding electronic diffraction micrographs. (A) Electron microscope image of gold triangular nanoparticles (AuTNPs) prepared using $40 \mu \mathrm{L}$ of lemongrass extract (LG Ext) in $1 \mathrm{~mL}$ of $1 \mathrm{mM}$ of $\mathrm{HAuCl}_{4}$ solution showing triangular nanoparticles. (B) The selected area electron diffraction pattern (SAED) of the AuTNPs showing the circled, triangular and cubic spots, which are corresponding to $1 / 3\{422\},\{200\}$ and $\{311\}$ Braggs reflections, respectively. (C) TEM image of triangular silver (AgTNPs) prepared by using $20 \mu \mathrm{L}$ of $\mathrm{Ag}$ seeds, and (D) SAED pattern showing the circled, triangular and cubic spots, which are corresponding to $1 / 3\{422\},\{200\}$ and $\{311\}$ Braggs reflections, respectively. (E) TEM image of spherical gold nanoparticles (AuSNPs) prepared using $80 \mu \mathrm{L}$ of LG Ext in $1 \mathrm{~mL}$ of $1 \mathrm{mM}$ of $\mathrm{HAuCl}_{4}$ solution and $(\mathrm{F})$ its corresponding electronic diffraction pattern showing (111), (200), (220) and (311) Face centered cubic structure (FCC) planes. (G) TEM image showing spherical silver (AgSNPs) formed using $30 \mu \mathrm{L}$ of $\mathrm{Ag}$ seeds and $(\mathrm{H})$ its corresponding electronic diffraction pattern, showing indexed ring pattern of (111), (200), (220) FCC planes. A representative nanoparticle of each class is pseudo colored based on the color observed from the nanoparticle solution. 

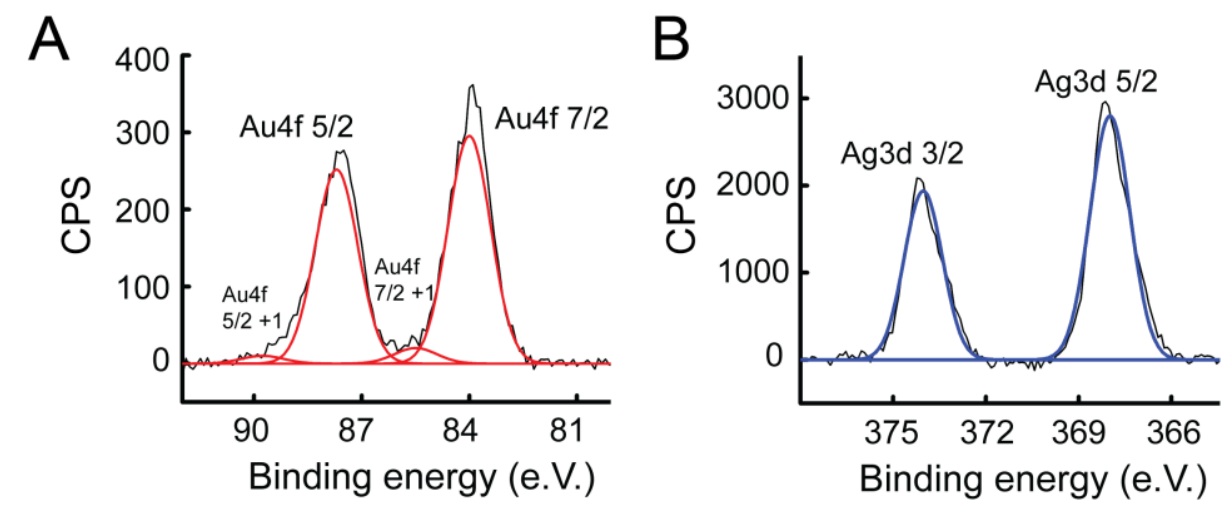

Figure S4. X-ray photoelectron spectroscopy (XPS) analysis of synthesized nanoparticles illustrating the reduction potential of reducing agents. Counts per second (CPS) is plotted as a function of binding energy (measured in electron volts). (A) The spectra of gold nanoparticles synthesized by using lemongrass extract as a reducing agent, and XPS spectra are recorded after 24 hours of completing the reaction. The spectra showing Au $4 \mathrm{f}$ core level spectrum of for AuNPs (Au4f 5/2) and (Au 4f 7/2). The doublet peaks at 87.7 and $84.0 \mathrm{eV}$ correspond to $\mathrm{Au} 4 \mathrm{f} 5 / 2$ and $4 \mathrm{f} 7 / 2$, respectively, denoting $\mathrm{Au}(0)$ oxidation state assignment, where peaks at $86.0 \mathrm{eV}$ and $89.7 \mathrm{eV}$ are associated with $\mathrm{Au}(+1)$ oxidation state. The spectrum obtained (black) is fitted (red) in Origin Pro 9.0 software. (B) Blue colored spectrum is the fitted spectra of silver nanoparticles synthesized by chemical method and XPS recorded after 24 hours of completing the reaction, showing Ag $3 \mathrm{~d}$ core level spectrum of AgNPs (Ag 3d 3/2) and (Ag 3d 5/2). The peaks at 368.2 and $374.2 \mathrm{eV}$ correspond to $\mathrm{Ag}$ $3 \mathrm{~d} 5 / 2$ and $3 \mathrm{~d} 3 / 2$, respectively for the $\mathrm{Ag}(0)$ oxidation state. The spectrum obtained (black) is fitted (blue) in OriginPro 9.0 software. The peak assignments and background correction have been performed using OriginPro 9.0 software. 
A

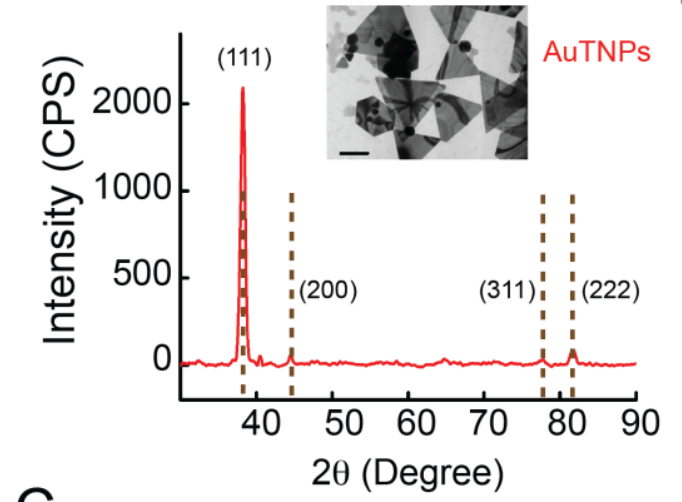

C

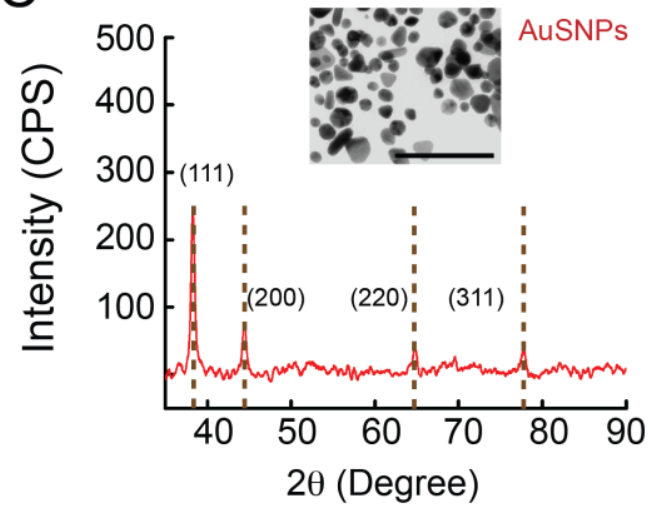

B
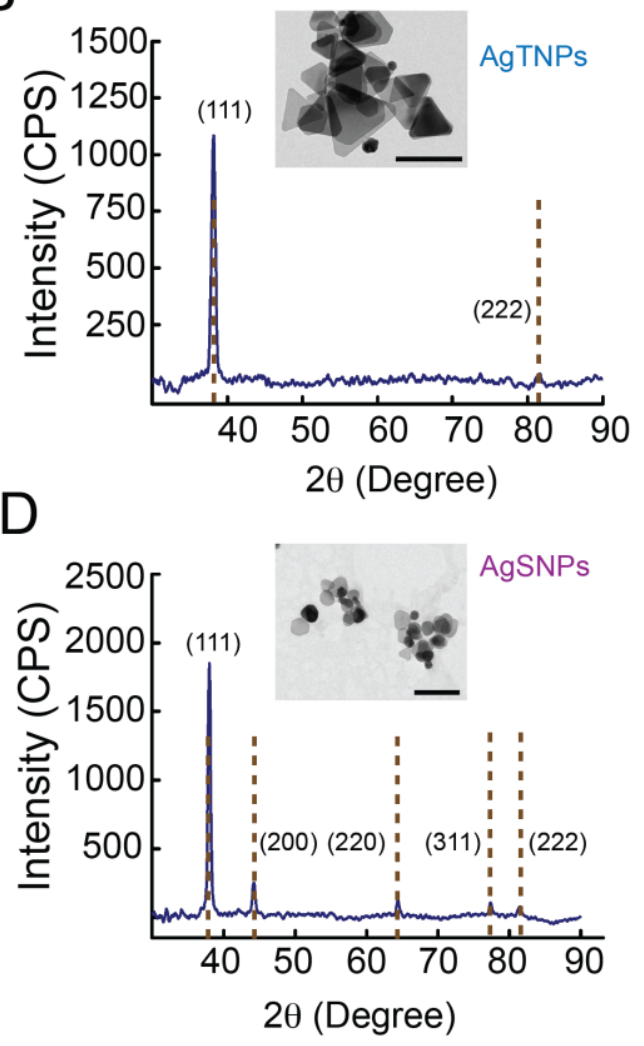

Figure S5. X-ray diffraction (XRD) analysis of nanoparticles. X-ray diffraction data of triangular and spherical $\mathrm{Au}$ and $\mathrm{Ag}$ nanoparticle showing spacing of the crystal packing of the nanoparticle. Inset showing the corresponding transmission electron microscopy (TEM) images of silver (AgNPs) and gold (AuNPs) nanoparticles. Scale bar is $200 \mathrm{~nm}$. XRD of (A) gold triangular nanoparticles (AuTNPs) showing preferred (111) plane with low intense FCC plane (200). (B) XRD of silver triangular nanoparticles (AgTNPs) showing preferred (111) plane, with low intense and broadened other FCC planes (200), (220), and (311). (C) XRD of gold spherical nanoparticles (AuSNPs) showing (111), (200), (220) and (311) planes and (D) silver spherical nanoparticles (AuSNPs) showing (111), (200), (220) and (311) planes corresponding to FCC crystal lattice. Inset TEM image of corresponding diffraction pattern, respectively. 
A

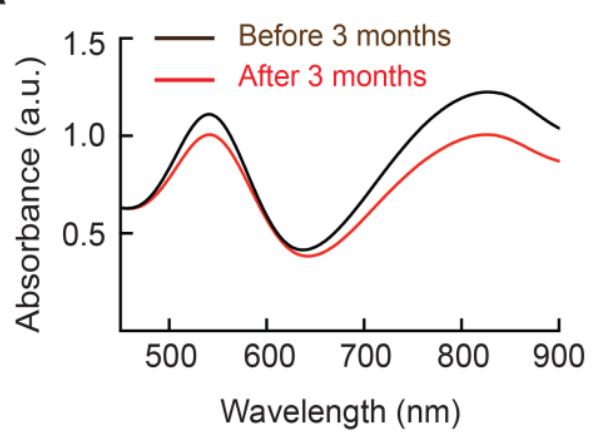

B

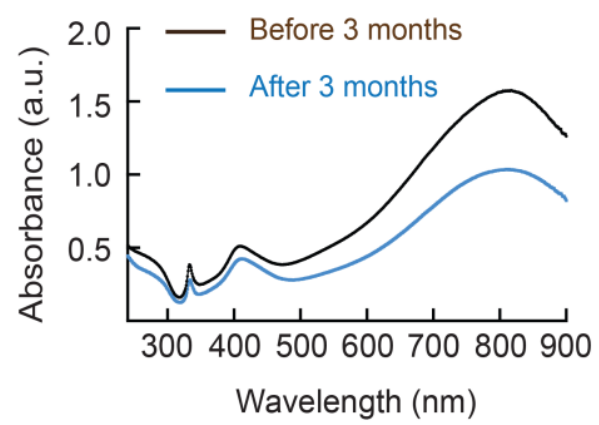

Figure S6. UV-Vis spectroscopic measurement of triangular gold (AuTNPs) and silver nanoparticles (AgTNPs). UV-Vis spectrum of (A) AuTNPs and (B) AgTNPs, recorded before and after 3 months of incubation at room temperature showing their stable nature over time.

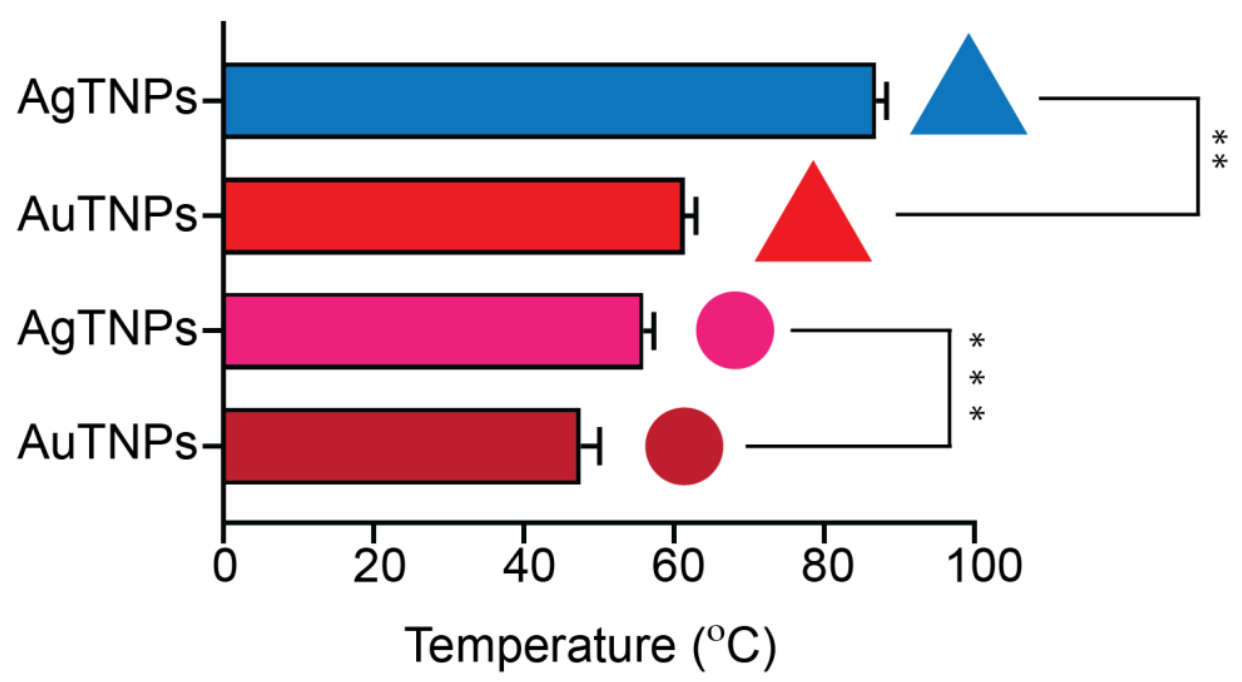

Figure S7. Photothermal light to heat conversion efficiency of nanoparticles recorded after irradiating nanoparticles at $808 \mathrm{~nm}$ NIR laser (1.2 Ampere, $120 \mathrm{~s}$ ). At similar concentrations $(0.4 \mathrm{mM})$, silver triangular nanoparticles (AgTNPs) attain higher temperatures upon irradiation with photonic source as compared to gold triangular nanoparticles (AuTNPs). The spherical silver (AgSNPs) and spherical gold nanoparticles (AuSNPs) attain comparatively lower temperature. The data represented are mean \pm standard deviation. Statistical significance was calculated using Students t-test and the p-values (**) are 0.0014 and (***) are $<0.0001$, respectively. 

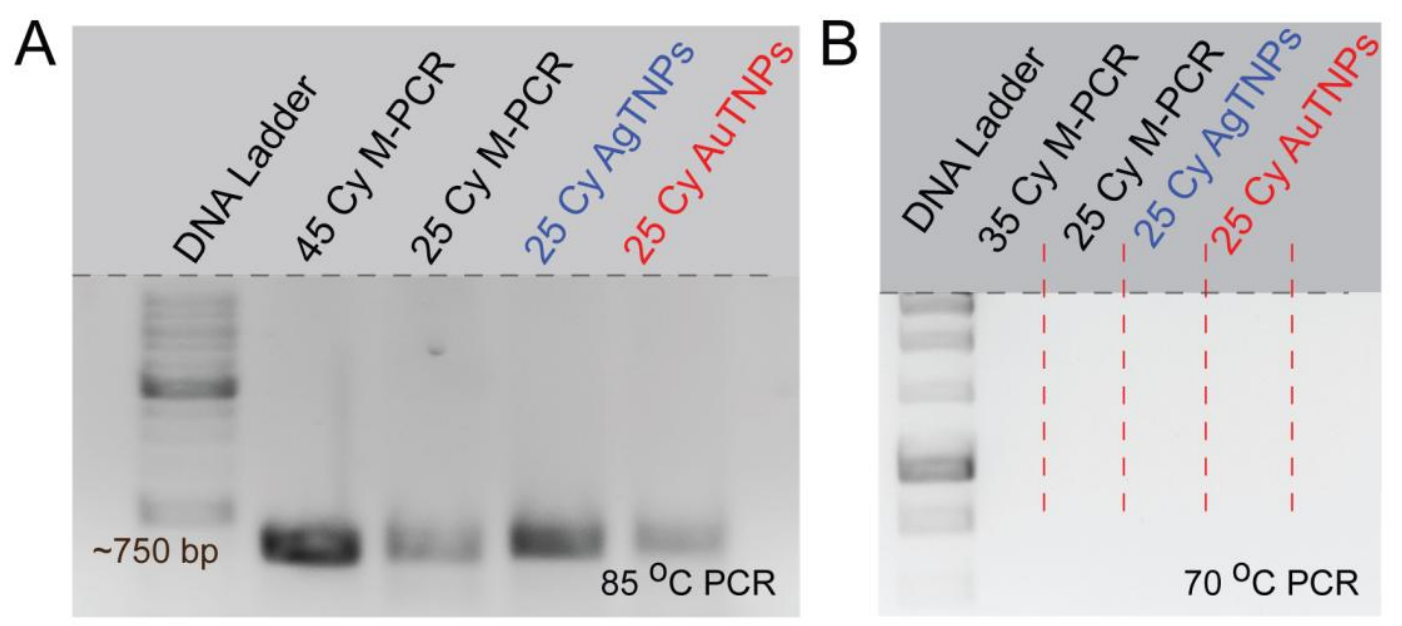

Figure S8. PCR amplification assay. Agarose gel electrophoresis analysis after performing triangular nanoparticles mediated photothermal PCR and Machine PCR as a control. (A) Agarose gel electrophoresis image of PCR products after 45 cycles of commercial machine PCR (45 Cy M-PCR), 25 cycles of commercial machine PCR (25 Cy M-PCR), 25 cycles of laser assisted machine-free PCR in presence of triangular gold nanoparticles (AuTNPs) and triangular silver nanoparticles (AgTNPs) at $85^{\circ} \mathrm{C}$. Machine-free PCR in presence of AgTNPs and AuTNPs produced similar yields to $25 \mathrm{Cy}$ M-PCR and AgTNPs yield was comparatively higher at $85{ }^{\circ} \mathrm{C}$. (B) PCR products after 35 cycles (Machine PCR), 25 cycles of machine, AuTNPs and AgTNPs at $70{ }^{\circ} \mathrm{C}$ showing no PCR amplification.
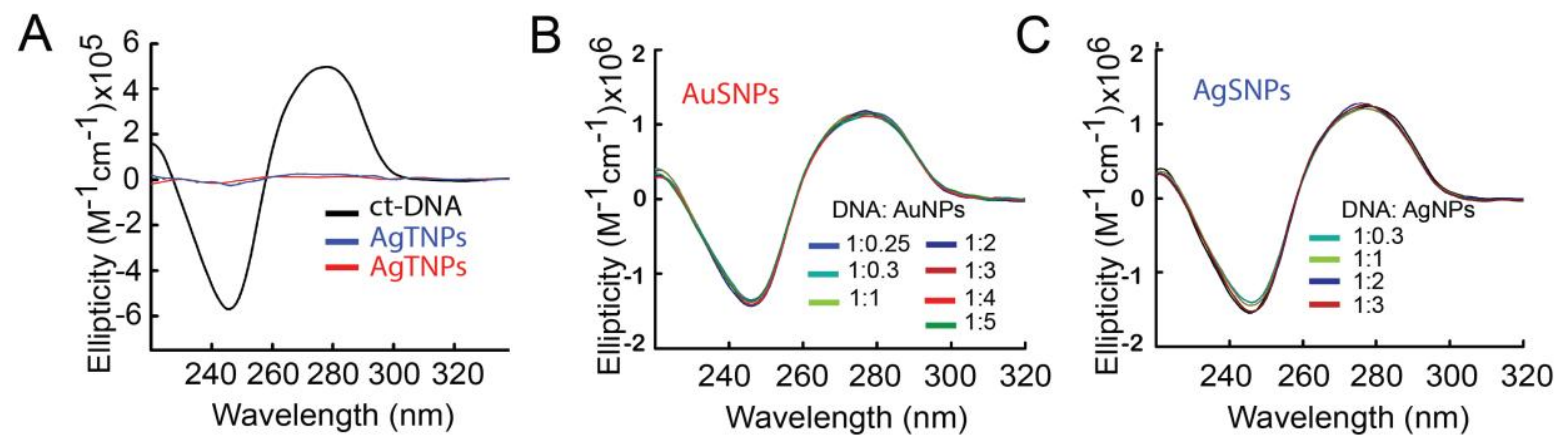

Figure S9. Circular dichroism (CD) analysis of gold and silver triangular nanoparticles and binding with Ct-DNA, by keeping Ct-DNA concentration constant $(600 \mu \mathrm{M})$ and varying concentration of nanoparticles. (A) CD spectrums of ct-DNA, triangular silver (AgTNPs) and triangular gold nanoparticles (AuTNPs) AuTNPs and AgTNPs are showing no signal in CD spectrum. (B) Ct-DNA: gold spherical nanoparticles (AuSNPs) with increasing concentration ratio of NPs (1:0.25-1:5) showing no promising change in the CD spectrum. (C) Ct-DNA: spherical silver nanoparticles (AgSNPs) with concentration ratio of NPs (1:0.3-1:3) showing no significant change in the spectrum. 


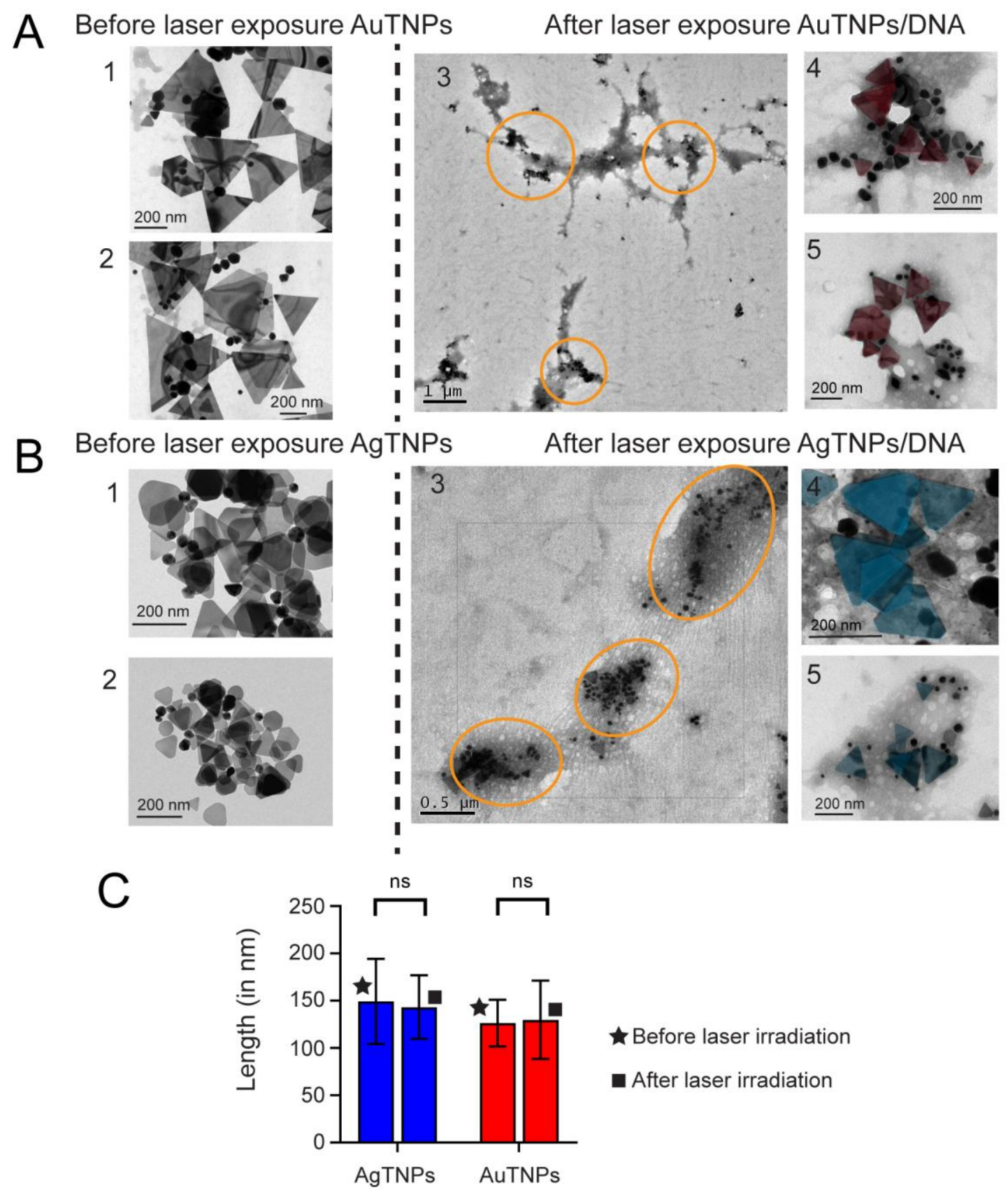

Figure S10. Transmission electron microscopy (TEM) results of triangular nanoparticles (TNPs) before and after $808 \mathrm{~nm}$ laser irradiation with DNA/PCR mixture. Figure A (panels 1-2) micrographs recorded before laser irradiation showing high surface to volume ratio of triangular gold nanoparticles with minor spherical particles, and Figure A (panels 3-5) micrographs recorded after 35 thermal cycles showing nanoparticles intact with DNA/PCR mixture. Similarly, Figure B (panels 1-2) micrographs recorded before laser irradiation showing high surface to volume ratio of triangular silver nanoparticles (AgTNPs) with minor spherical particles, and Figure B (panels 3-5) micrographs recorded after 35 thermal cycles showing nanoparticles intact with DNA/PCR mixture. Figure $\mathrm{C}$ showing measure of edge length of triangular nanoparticles before and after laser irradiation. NS denotes nonstatistically significant changes in the particles. 
Table S1. Laser unit conversion table: Ampere (A) to milliwatt (mW)

\begin{tabular}{|c|c|}
\hline $\begin{array}{c}\text { Laser Input } \\
\text { values in } \\
\text { Ampere }(\mathrm{A})\end{array}$ & $\begin{array}{c}\text { Power } \\
(\mathrm{mW})\end{array}$ \\
\hline 0.2 & 0 \\
\hline 0.4 & 4 \\
\hline 0.6 & 210 \\
\hline 0.8 & 420 \\
\hline 1 & 644 \\
\hline 1.2 & 868 \\
\hline 1.4 & 1095 \\
\hline 1.6 & 1290 \\
\hline
\end{tabular}

\section{References}

[1] S. S. Shankar, A. Rai, A. Ahmad, M. Sastry, N. Group, M. Chemistry, V. Di, B. Sciences, V. Di, Chem. Mater.2005, 17, 566-572.

[2] B. D. Aherne, D. M. Ledwith, M. Gara, J. M. Kelly, Adv. Funct. Mater.2008, 18, 2005-2016. 